\title{
LOAD DISTRIBUTION IN A MODEL OF A HIP JOINT
}

\author{
H. Fessler, NotTingham, ENGLAND
}

From the Department of Mechanical Engineering, Nottingham University

The distribution of load through bone and joints is of interest to anatomists and orthopaedic surgeons. The first study of this problem was published by Meyer (1867). In that paper it was shown that the arrangement of the trabeculae in the upper part of the femur was similar to the distribution of the stress trajectories in a curved cantilever, called a crane by Culmann, the engineer who calculated the stress trajectories. Wolff (1870) repeated Meyer's work with several specimens and better bone section. The approximate correspondence between the networks of trabeculae and stress trajectories was the foundation of the theory of the functional structure of bones.

Koch (1917) completely sectioned a femur and calculated the compressive, bending and shearing stresses that act on each section. He thus laboriously carried out, for the irregular shape of the actual femur, the same calculations that Culmann had made for a simplified shape, and confirmed the functional theory of bone structure. Thus it is now generally accepted that the final shape and intimate arrangement of the bone will depend on the nature of the forces acting on it. The analysis of these forces continues to be of importance in determining at least some of the factors that ultimately condition the behaviour of the bone and perhaps even its pathology (Trueta 1953, Harrison, Schajowicz and Trueta 1953, Trueta 1954).

The terms "femur" and "pelvis" will be used to describe the parts of the models as well as the actual bones. The photoelastic techniques which were employed in this study will be briefly described. Physical terms which may be unfamiliar to some readers are explained in an appendix at the end of the paper.

\section{THE PHOTOELASTIC METHOD}

Photoelasticity (Jessop and Harris 1949) is based on the double refracting properties of certain plastics. If a model is cut from a flat plate of such a material and forces are applied to it in the plane of the plate, two types of optical interference patterns may be observed when the model is examined in polarised light.

With plane polarised light no light is transmitted through parts of the model where the directions of the principal stresses are parallel to the direction of polarisation. By successive variation of the direction of polarisation it is therefore possible to plot the isoclinics (lines of equal inclination of principal stresses) for a number of directions. The lines of action of the principal stresses, called "stress trajectories," are derived from the isoclinics.

The maximum shear stress (or difference of principal stresses) at any point in the model is proportional to the relative retardation of the two component rays of polarised light that pass through the point. When the relative retardations are equal to an integral number of wave lengths of the light used, no light is transmitted. It follows that, in monochromatic light, lines of constant shear stress appear as dark bands, called "fringes." When the calibration constant of the material is known, the stresses may be determined from the fringe orders and isoclinics at the required point. Fringes are drawn in Figure 3 and the numbers indicate their order; thus the maximum shear stress near line 6 is six times that in the vicinity of line 1 , and the regions beyond the line 0 are almost stress free.

\section{EXPERIMENTAL TECHNIQUES}

Because this type of photoelastic analysis is limited to flat plates, the frontal plane, which contains the highest point of the head of the femur, was investigated. It was assumed that this plane would contain the point of maximum pressure.

VOL. 39 B, No. 1, FEBRUARY 1957 
Plaster casts of the relevant parts of a normal pelvis and femur were set up in the normal, upright position and cut along the required plane. Photoelastic models a quarter of an inch thick were made identical to these sections. The material used was Araldite Casting Resin B, an ethoxylene resin (Spooner and McConnell 1953). The chain-dotted lines in Figure 1 show the shape of the bones and the full lines indicate the shape of the models. Near the neck

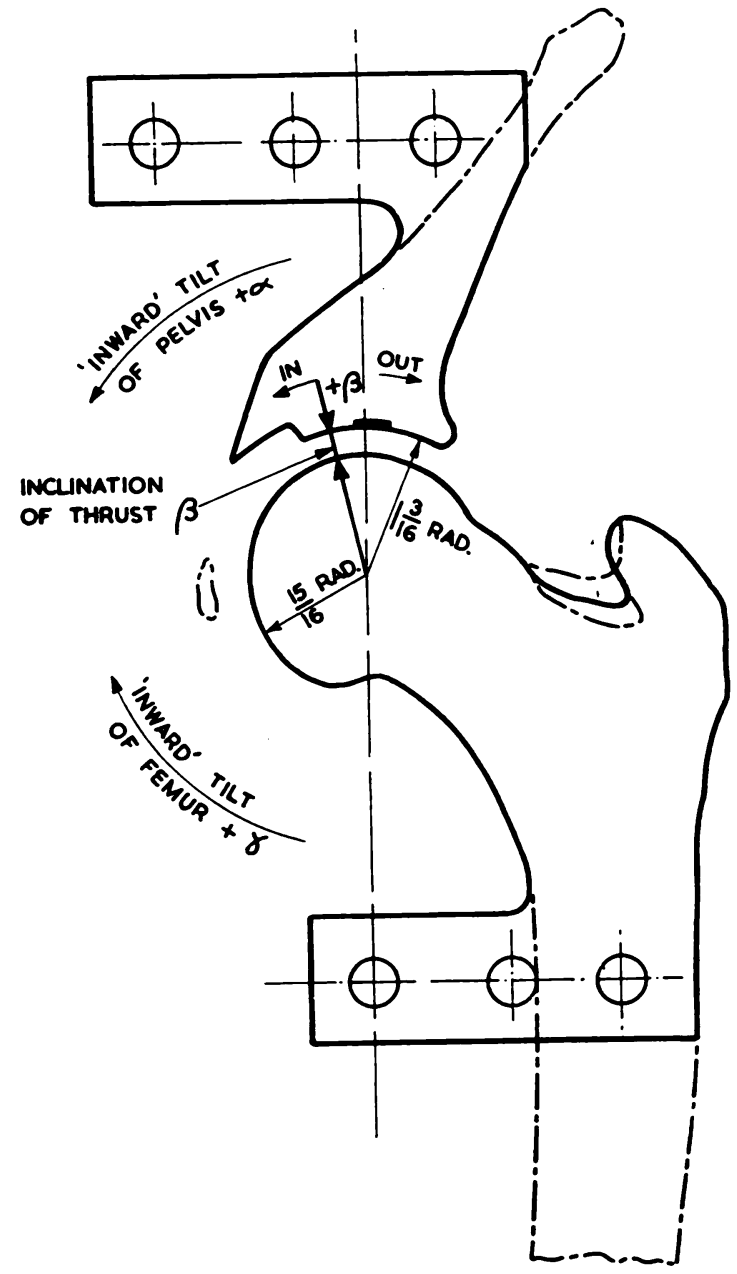

Fig. 1

Models of bones and definition of positions of bones and inclination of thrust. The thick line on the acetabular roof shows the movement of the point of maximal thrust caused by inclinations of the pelvis from $\alpha=+20$ degrees to $\alpha=-20$ degrees.

of the femur the shape of the model was a compromise between the actual section considered and the strongest section of the bone. The lugs and holes were necessary for the application of the test loads.

The space between the bones was a quarter of an inch and this space between the models had to be filled by an equivalent to cartilage. Strips of rubber were used for this purpose.

A lever type straining frame was constructed in such a way that the "pelvis" was constrained to move vertically (without tilting) under the action of a vertical force, while the "femur" was clamped to a fixed part of the frame. The load could be arranged to act 
through the centre of the head of the femur or could be displaced inwards or outwards from that position. Both " bones" could be clamped in their normal or inclined positions. This apparatus is shown in Figure 2 mounted across the polariscope.

Weights placed in the scale pan of the straining frame (not shown in Figure 2) caused a compressive force to be transmitted from the pelvis through the rubber packing to the femoral head. The resulting fringe and isoclinic patterns could be observed by viewing through the analyser seen at the right of Figure 2. Records were taken by projecting and tracing the

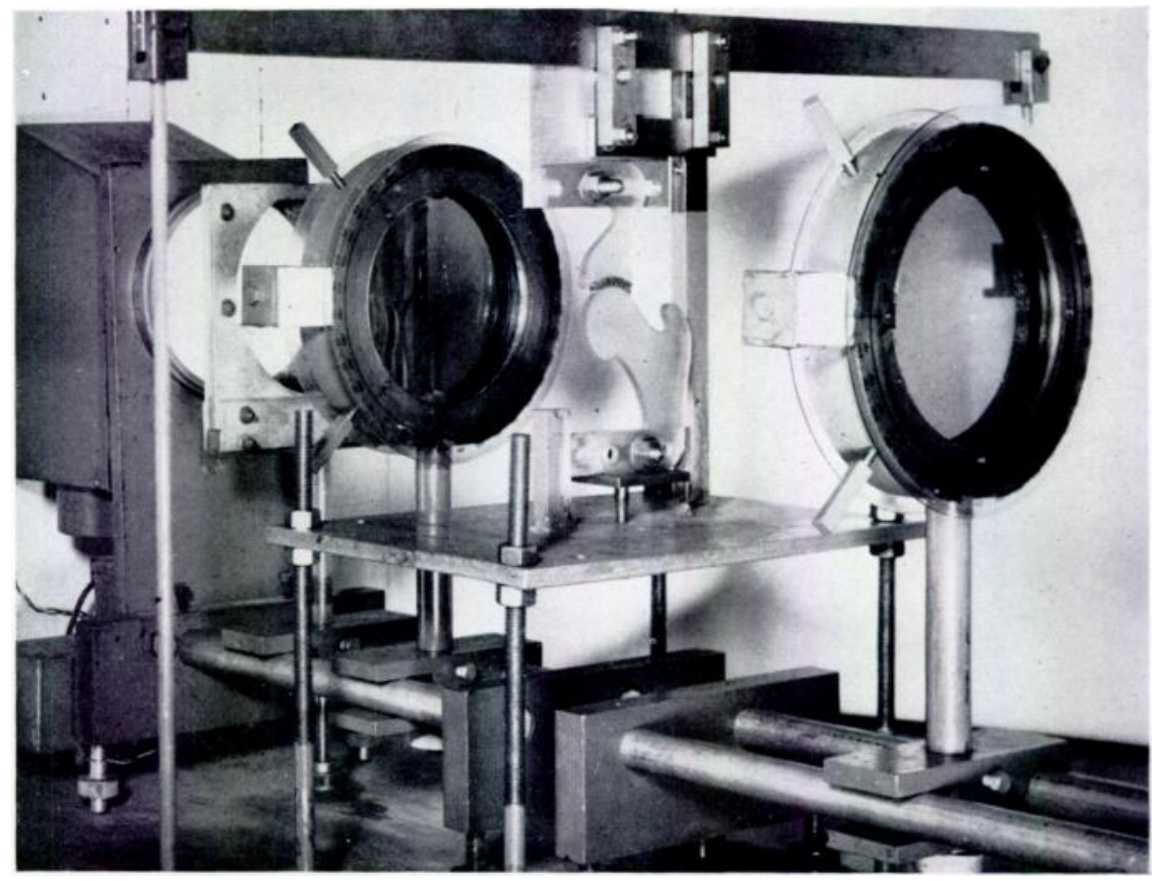

Fig. 2

View of apparatus.

resulting patterns, which were enlarged to twice natural size. A typical fringe pattern is shown in Figure 3. The lines are loci of constant maximum shear stress. Figure 4 is a photograph of the fringe pattern (for a smaller load than used for Figure 3).

The prime objective of this investigation was to study the load distribution on the acetabular roof-that is, to determine the compressive stress perpendicular to that surface. This compressive stress can be obtained only by a cumbersome process of graphical integration, based on the fringe and isoclinic patterns. This integration was carried out for the normal position, when the load acted through the centre of the femoral head, and it was found that the position of the maximum compressive stress coincided with the position of maximum shear stress (or maximum fringe order). Because all the fringe patterns were very similar it was assumed that this coincidence was general.

The numerical value of the stress at any point in the model is of little interest because the model consists of flat plates which carry the whole load, whereas the central section of the actual spherical joint carries only an unknown fraction of the load. Therefore the results cannot be directly related to the pressure on the bone surface. However, it seems a reasonable assumption that the position of the peak stress on the sphere will coincide with that on the central frontal section which was studied.

VOL. 39 B, NO. 1, FEBRUARY 1957 
The position of the peak stress for different conditions was therefore determined by plotting the fringe order at the edge of the pelvis. Because both surfaces are spherical it is convenient to express position by the angle between the vertical and the normal to the surface. These normals are radial lines from the centre of the femoral head. A typical graph, as derived from Figure 3, is shown in Figure 5.

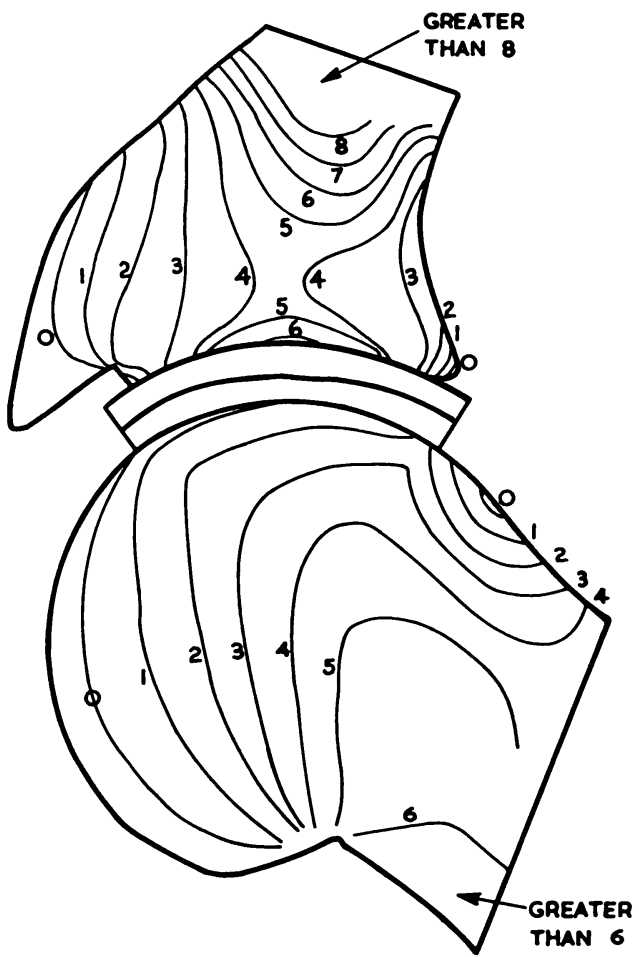

Fig. 3

Typical fringe pattern. The lines are loci of constant maximal shear stress and the numbers indicate the relative magnitude of this stress.

\section{RESULTS}

In the first series of tests both bones were maintained in their normal positions and the vertical line of action of the applied force or load was displaced horizontally relative to the bones. It was found that the fringe distribution on the acetabular roof was unaffected by this variation and that the maximum thrust always occurred through the highest point of the femoral head. The load was arranged to act through the centre for all further tests.

In the second series the femur was inclined while the pelvis remained in the normal position. It appears from Figure 5 that inward inclination of the femur has little effect on the load distribution on the acetabular roof. The inclinations are as defined in Figure 1.

In the third series the femur was maintained in its normal position while the pelvis was tilted. It is seen from Figure 7 that when the inward inclination of the pelvis exceeds 5 degrees an "inward" movement of the point of maximum pressure occurs. It was assumed that the resultant force on the joint acted along the line through this point and the centre of the femoral head. This was justified because all the fringe order graphs were approximately symmetrical about the peak value. It follows from this assumption that an "inward" movement of the point of maximum thrust indicates a resultant inward thrust on the pelvis. The reaction to this force is an outward force on the femur-that is, a force tending to push 
the head of the femur out of the socket. This outward force is conveniently represented as a fraction of the vertical force on the joint, i.e., by the trigonometric ratio " $\tan \beta$," where $\beta$ is the angle of inclination (relative to the vertical) of the maximum thrust. This quantity is plotted in Figure 8 for inclination of the pelvis (femur fixed in normal position $\diamond=0$ ) and for equal inclinations on both bones. It is seen that the clinically important outward force is not significantly increased by the rotation of the femur.

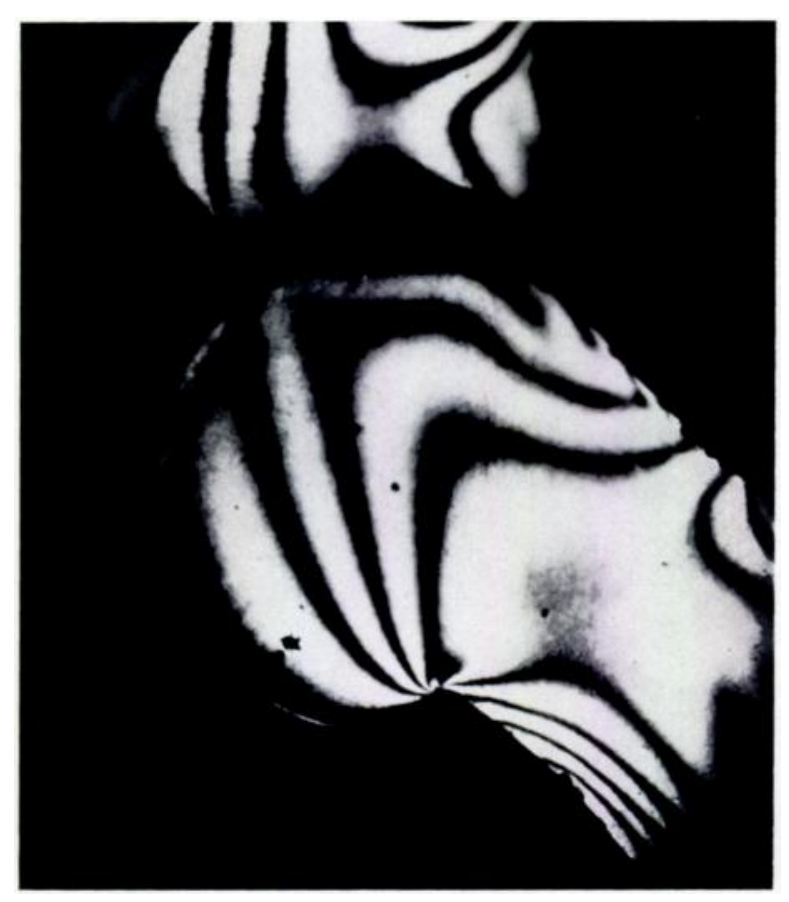

FIG. 4

Photograph of fringe pattern.

Figure 9, which is also derived from Figure 7, shows the movement of the point of maximum thrust on the acetabular roof. This is indicated by a thick line in Figure 1. It is to be noted that outward tilting of the pelvis causes only slight inward movement of the thrust. Inward inclination of 15 degrees or more of the pelvis causes the point of maximum pressure to move 8 degrees outwards. This represents a movement of 4 millimetres or 37 per cent of the distance from the normal, vertical, point of maximum thrust to the outer edge of the acetabulum, an appreciable disturbance.

The stress trajectories, or lines along which the stresses act, were derived from the isoclinic pattern and are shown for the normal position in Figure 10. It must be emphasised that these lines indicate directions only of the principal stresses and are independent of their magnitude. Their arrangement agrees with that calculated for an actual femur by Koch (1917). The similarity of the pattern of trabeculae to Figure 10 is taken to justify the use of photoelastic models in the study of bone problems. The agreement between Figure 10 and Koch's pattern also shows that the assumptions of elementary strength of materials, on which Koch's calculations are based, do not introduce any appreciable errors in this case.

\section{APPLICABILITY OF RESULTS}

The load distribution on the joint is a function of the physical properties of the components as well as their geometry. When homogeneous, isotropic bodies are in contact, the load 


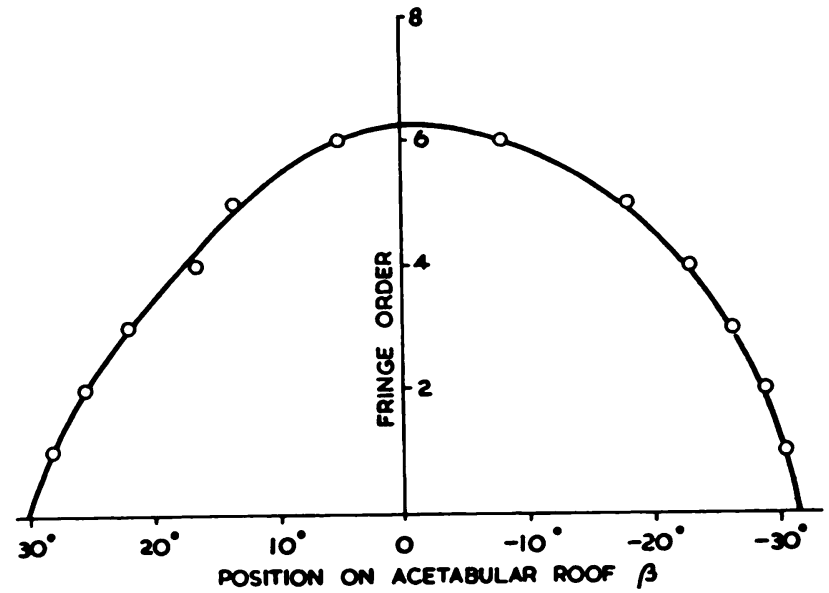

Fig. 5

Distribution of shear stress on acetabular roof, obtained from the fringe pattern of Figure 3.

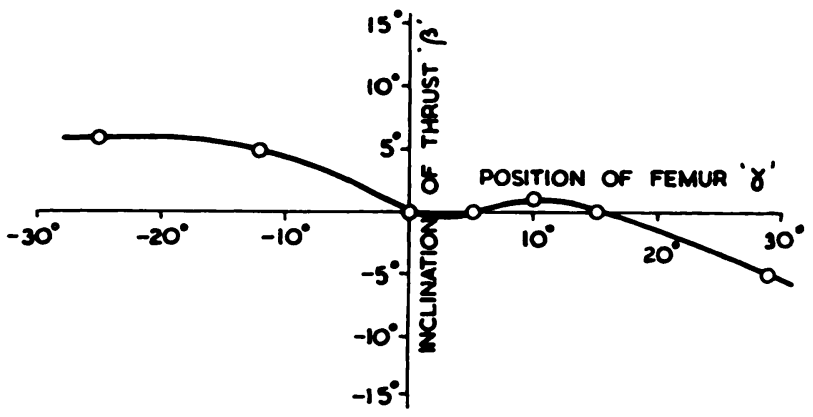

Fig. 6

Effect of inclination of femur; $\beta$ and $\varnothing$ are measured from the vertical direction.

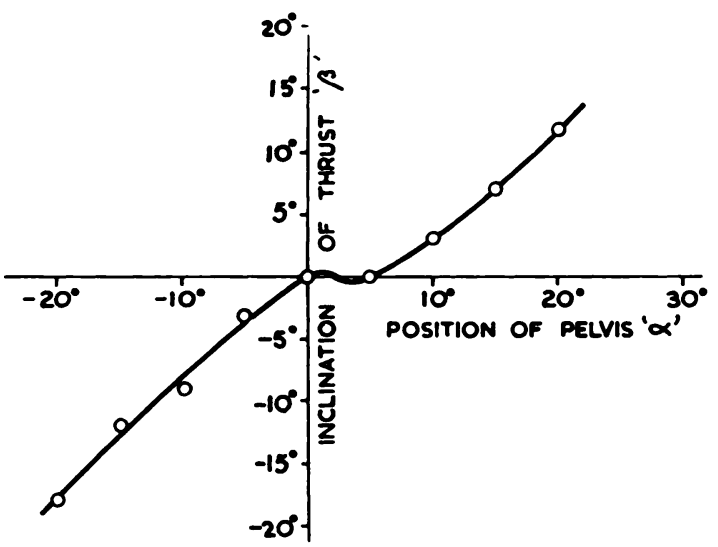

FIG. 7

Effect of inclination of pelvis; $\alpha$ and $\beta$ are measured from the vertical direction.

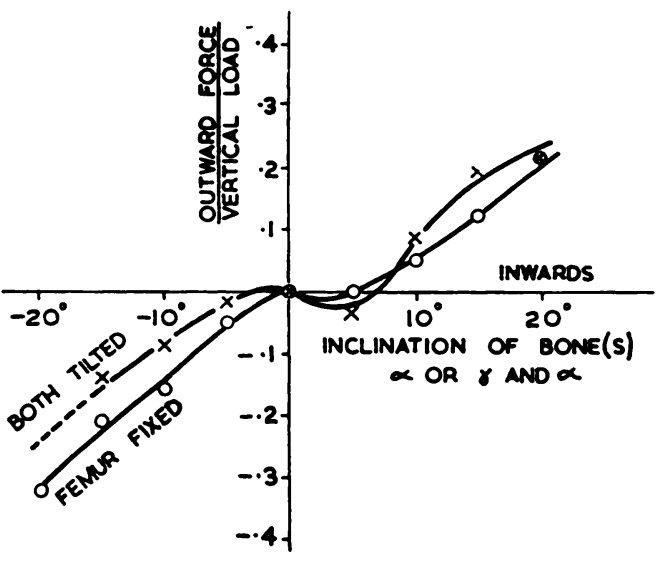

Fig. 8

Lateral force due to inclination. The inclinations of the bones are measured from the vertical. $O$ represent tilting of pelvis only but $X$ represent simultaneous tilting of pelvis and femur.

THE JOURNAL OF BONE AND JOINT SURGERY 
distribution depends on their elastic moduli (stress/strain) and their values of Poisson's ratio (strain perpendicular to direction of loading/strain in the direction of loading). The effect of the latter is usually small. As neither bone nor cartilage is a homogeneous

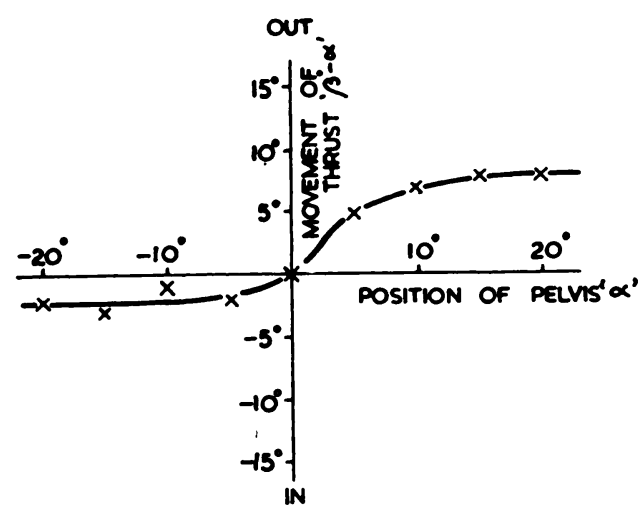

Fig. 9

Movement of point of maximal thrust on acetabular roof; $\alpha$ is measured from the vertical, but $(\beta-\alpha)$ is measured from the (normal) apex of the acetabulum.

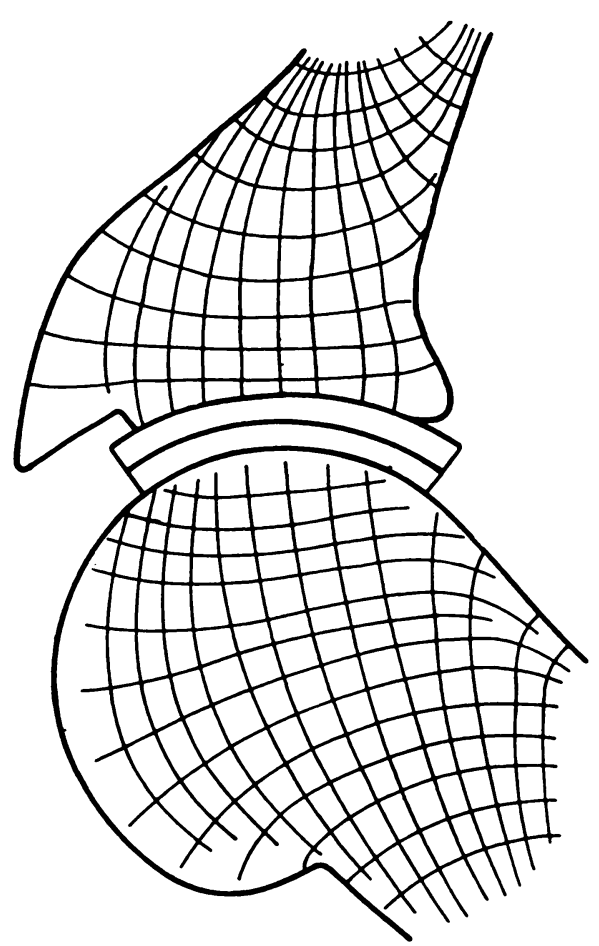

Fig. 10

Stress trajectories. The lines indicate the directions only of the principal stresses, compression along the vertical lines and low stresses along the horizontal lines.

isotropic substance the only significant fact is that the latter is much softer than the former.

Values of elastic moduli have been reported (Jakobsson 1954) as 200,000 kilograms/square centimetre for bone and 100 kilograms/square centimetre for cartilage. These average values 
indicate that, under the same load, the compressive strain of the cartilage is 2,000 times that of the bone; this indicates that the bones do not change in shape under normal loading.

The elastic modulus of Araldite Casting Resin B, the ethoxylene resin used as the model material, is $530,000 \mathrm{lb}$./square inch (about 37,000 kilograms/square centimetre). It was not possible to reproduce the ratio of the elastic moduli of the actual joint because the packing which represented the cartilage would have been so soft that it would have been squeezed out of joint. The real cartilage is in the form of a curved sheet and therefore less liable to be squeezed out. Strips of rubber of modulus $6,500 \mathrm{lb}$./square inch (about 460 kilograms/square centimetre) were used. The ratio of the elastic moduli was therefore only 81 , but it was considered that errors due to this would be small compared with those due to lack of homogeneity and isotropy of the bones and cartilage.

\section{CONCLUSIONS}

Because of the above limitations and the fact that only one section of the joint was considered, the results are not directly applicable to actual bones, but they suggest the following conclusions.

1. Greater changes in the load distribution on the acetabulum are caused by inclination of the pelvis than by movement of the femur.

2. Inward inclination of the pelvis tends to force the femoral head out of its socket and moves the maximal thrust towards the outer edge of the acetabulum.

3. The load distribution on the joint is not significantly changed if the line of action of the load is moved relative to the joint.

4. The arrangement of the stress trajectories in the photoelastic model is similar to that of the trabeculae in the actual bone.

5. If certain assumptions are made, photoelastic tests may provide information about the stress distribution in bones as well as in joints.

\section{SUMMARY}

Photoelastic techniques were used to study the stresses in models which represented the central frontal plane of a normal hip joint. The pelvis and the femur were inclined and the direction of the resultant thrust on the joint was estimated. Inward tilting of the pelvis causes a force which tends to push the head of the femur out of joint.

The load distribution on the joint is not significantly changed if the line of action of the load is moved relative to the joint. The directions of the lines of principal stress in both bones are shown.

Mr J. P. Campbell, F.R.C.S., initiated this investigation and supplied the plaster casts of the bones. The author would also like to thank Professor J. Trueta for his interest and helpful suggestions. Professor J. A. Pope, Head of the Departments of Civil and Mechanical Engineering at Nottingham University, provided all necessary facilities for this work. Dr J. H. Fessler, M.A., made many suggestions on how the subject matter could best be presented to medical readers.

\section{APPENDIX}

Explanation of optical terms-Plane polarised light consists of light waves that vibrate in one plane only. This is obtained by passing ordinary light through an optical filter or polariser, usually a sheet of Polaroid. The direction of polarisation is perpendicular to the plane of polarisation. At any particular point in the model light waves are only transmitted in two mutually perpendicular directions. These directions are directions of the principal stresses (see later) at that point. The two mutually perpendicular waves travel through the model at different velocities so that a relative retardation exists between them after passage through the model. When this relative retardation is equal to an integral number of wave lengths (e.g., $0,1,2$, etc.) no light is transmitted through a second polariser, if the axis of the latter is perpendicular to the first polariser. If no light is transmitted, the particular point will form part of a dark region called a fringe. There is one fringe for each wave length of relative retardation and the number of wave lengths is called the fringe order. 
Explanation of mechanical terms-The intensity of a force is called a stress; it is equal to the magnitude of the force, divided by the area on which it acts. Corresponding to the different forces, one must distinguish tensile, compressive and shear stresses. If the direction of a force is inclined to the plane (or section) in the body which is being considered, it will cause a direct stress (tensile or compressive) and a shear stress on that plane. If the plane is perpendicular to the direction of the (tensile or compressive) force there will be no shear stress and the direct stress is called a principal stress. At every point in a body there are three such mutually perpendicular planes, and the directions at right angles to them are called the directions of principal stresses. On planes inclined at 45 degrees to the planes of principal stress the direct stress is zero and the shear stress has its greatest value. This is called the maximum shear stress. It is equal to half the difference of the principal stresses and it has been found that the fringe order is proportional to this quantity.

When a tensile force acts on a body it elongates it. The elongation divided by the original length is called the direct strain. This elongation in the direction of the force is always associated with a contraction perpendicular to the direction of the force, called lateral strain. The lateral strain divided by the direct strain is called Poisson's ratio, one of the fundamental properties of an isotropic material, which is unchanged if the force (and the resultant strains) is reversed.

The physical quantity used to describe how much a body elongates under the action of a tensile force (or compresses due to a compressive force) is the modulus of elasticity or Young's modulus. It is the stress divided by the strain and may be imagined to be the stress which would be necessary to cause elongation equal to the original length if the body did not break, but continued to extend in the same manner as under moderate stresses.

These explanations are as brief and as simple as possible. Fuller explanations and rigorous definitions may be found in any standard text-book of photoelasticity and strength of materials.

\section{REFERENCES}

Harrison, M. H. M., Schajowicz, F., and Trueta, J. (1953): Osteoarthritis of the Hip: A Study of the Nature and Evolution of the Disease. Journal of Bone and Joint Surgery, 35-B, 598.

Jakobsson, A. (1954): The Shelf Operation. An Evaluation of Results in Congenital Dysplasia, Subluxation and Dislocation of the Hip Joint. Acta Orthopaedica Scandinavica, Supplementum No. 15.

Jessop, H. T., and Harris, F. C. (1949): Photoelasticity: Principles and Methods. London: Cleaver-Hume Press.

КосH, J. C. (1917): The Laws of Bone Architecture. American Journal of Anatomy, 21, 177.

MeYer, H. (1867): Die Architectur der Spongiosa. Archiv für Anatomie, Physiologie und wissenschaftliche Medicin, p. 615 .

SPOONER, H., and MCConnell, L. D. (1953): An ethoxylene resin for photoelastic work. British Journal of Applied Physics, 4, 181.

TruetA, J. (1953): Recherches récentes sur la pathogénie de la coxarthrose. Mémoires de l'Académie de Chirurgie, 79, 427.

Trueta, J. (1954): Osteoarthritis of the Hip. Annals of the Royal College of Surgeons of England, 15, 174. WolfF, J. (1870): Ueber die Innere Architectur der Knochen und ihre Bedeutung für die Frage vom Knochenwachsthum. Virchow's Archiv für pathologische Anatomie und Physiologie, 50, 389. 